monitoring of treatment response, understanding mechanisms of disease, and facilitating patient understanding of the condition will be explored. Disclosure of Interest: N. Dalbeth Consultant for: Kowa, Horizon DOI: 10.1136/annrheumdis-2018-eular.7767

\section{SP0069 IMPROVING ADHERENCE IN GOUT THERAPY}

\author{
A. Abhishek. Academic Rheumatology, The University of Nottingham, Nottingham, \\ UK
}

Gout is the commonest inflammatory arthritis and affects between $2.5 \%$ and $3.9 \%$ of the adult population in the western world, with even higher prevalence estimates reported elsewhere. It is the only form of arthritis that can be "cured" with simple, inexpensive pharmacologic urate lowering treatment. Such medicines offer the potential for "cure" to most people with gout. However, despite this, gout patients have the lowest adherence to long-term pharmacologic treatment among people with different rheumatic diseases. The reasons for this are multifactorial but include misconceptions about the disease and its treatment among patients, physicians and the wider community.

The long-term management of gout is overseen by primary care physicians in most countries. Recent studies have explored alternate models of gout managment and care, such as by community pharmacists and nurses. Such interventions have associated with a high uptake of urate lowering treatment and excellent persistence with treatment for upto five years.

This lecture will summarise the key barriers to the uptake of and adherence with urate lowering treatment. Recent randomised controlled trials and pilot studies examining the role of nurses and pharmacists in the management of gout using a treat-to-target approach will be discussed. New data about the mechanism by which such interventions may promote adherence with urate lowering treatment will be presented.

Disclosure of Interest: A. Abhishek Grant/research support from: AstraZeneca, Ironwood, Oxfordlmmunotech, Speakers bureau: Menarini

DOI: 10.1136/annrheumdis-2018-eular.7791

\section{SP0070 WHAT ARE GOUT GUIDELINES GOOD FOR ?}

\section{T. Bardin. Rheumatology, hôpital Lariboisière, Paris, France}

Guidelines aim to help physicians to make decisions in daily practice for an individual patient with a given condition. Guidelines should therefore be clear, easily readable and understandable by all physicians involved in disease care. They should also be as simple and brief as possible to be easily memorised by physicians, and this is a real challenge when guidelines aim at a full coverage of gout management. Most importantly, they cannot be taken as strict rules with legal implications. The final decisions concerning an individual patient remain in the hands of the responsible physician.

Guideline methodology has improved over years but still varies across recently published works. Ad hoc committees regularly involve all specialities involved in the disease care, and in the case of gout, general practitioners, who take care of most gout patients are now included but their number vary. Patients are not always included, despite being the final target of guidelines. Conflicts of interest of participating physicians are taken into account to a varying extent. The guideline development process involves an evaluation of all evidence available at the time of writing. Treatment impact on outcome, and assessment of drug benefit ratios are unanimously considered as important in the elaboration of guidelines. Factors such as drug pricing, availability and local regulatory agency recommendations, for instance about reimbursement, also matter, as guidelines should be practical in order to help physicians, but are rarely taken into account in guidelines.

There are several levels of evidence and the best ones, such as randomised placebo-controlled trials (RCT) or RCT meta-analyses are not available in all aspects of gout management. A large part of the published guidelines on the management of gout therefore relies on expert opinion, which remains fragile and may vary from one group to the other.

The numerous guidelines presently available for gout management exhibit more or less striking differences, and this diversity does not help guidelines to reach their goal. In this regard, the major differences observed between the simple and short guidelines issued by the American College of Physicians and the usually more complicated recommendations from the Rheumatology societies are the most detrimental. Pursuing therapeutic research on gout management and improving guideline methodology appear as sine qua non conditions to reach consensus and improve gout management.

Disclosure of Interest: T. Bardin Consultant for: astraZeneka, ipsen menarini, novartis

DOI: 10.1136/annrheumdis-2018-eular.7823
THURSDAY, 14 JUNE 2018

"Why does BMI matter?"

SP0071 IS OBESITY A RISK FOR RA?

T. Uhlig. Rheumatology, Diakonhjemmet Hospital, Oslo, Norway

According to the World Health Organisation (WHO) guidelines, individuals with a BMl of $>30 \mathrm{~kg} / \mathrm{m}^{2}$ are classified as obese. Obesity is on a global scale rising in prevalence and is a recognised as a cause of chronic disability. As is threatens public health, obesity has attracted significant attention in the general population, especially due to its association with significant metabolic and cardiovascular complications.

In research on rheumatoid arthritis (RA) BMI is frequently reported as a demographical variable, but obesity has not been thoroughly studied. A link between obesity and RA is plausible, as in adipose tissue biologic mechanisms of inflammation exist and may be linked to chronic systemic inflammation.

Obesity has in performed studies been considered a controversial risk factor for RA. While several studies have examined the potential influence of obesity on the development of RA, the results have been inconsistent.

While a few studies from the United States have found no or only modest association between obesity and the risk of RA, some European studies found significant risks for an association between RA and obesity. The prevalence of obesity has risen sharply in recent years which may increase our ability to better study possible associations between obesity and RA in the future. Existing evidence may thus point toward a slightly increased of obesity for the risk of RA.

Disclosure of Interest: None declared

DOI: 10.1136/annrheumdis-2018-eular.7812

\section{SP0072 DOES OBESITY INFLUENCE RA OUTCOMES ?}

P.D. Kiely. Rheumatology, St Georges University Hospitals NHS Foundation Trust, London, UK

The prevalence of obesity at diagnosis of rheumatoid arthritis (RA) has doubled in N. European and N. American populations from $\sim 14 \%$ ten years ago to $\sim 30 \%$ now. Several prospective studies of patients with early RA, treated with cSDMARD regimes, have found an association of baseline obesity with worse short and long term outcomes including DAS28 remission, low DAS, HAQ, pain assessed by VAS and SF36 physical and mental components. The most recently published cohorts report O.R. of obese RA patients at baseline achieving DAS28 remission of $0.71(0.55-0.93)$ at 2 years (versus non obese) and $0.53(0.39-0.71)$ at 3 years (versus normal BMI). A variety of composite responses (DAS28, CDAI, SDAI, EULAR) to TNF inhibitors, whether given in fixed dose s.c. or weight dosed i.v. formulations are significantly worse in obese RA patients from trial and registry data. In contrast the DAS28 response to Rituximab in RA patients is not affected by BMI, and ACR responses in Abatacept and Tocilizumab treated RA patients, whilst numerically less good in obese patients, are not disadvantaged by fixed s.c. dosing in comparison to i.v. dosing adjusted by weight.

Obesity is an increasingly prevalent co-morbidity and is associated with worse outcomes, including DAS28 remission, HAQ and pain scores.

Disclosure of Interest: P. Kiely Speakers bureau: BMS, Pfizer, Roche DOI: 10.1136/annrheumdis-2018-eular.7756

\section{THURSDAY, 14 JUNE 2018 \\ Musculoskeletal pain; feeding the opioid epidemic}

\section{SP0073 OPIOID PRESCRIBING: WHAT'S THE PROBLEM?}

B.H. Smith ${ }^{1,2}$, on behalf of Scottish Pain Research Community (SPaRC). ${ }^{1}$ Pain Service, NHS Tayside; ${ }^{2}$ Division of Population Health Sciences, University of Dundee, Dundee, UK

Opioids have been used for at least 7,000 years in the treatment of acute pain, to aid sleep and to promote euphoria. Their short-term benefits are well-recognised, but longer-term benefits are less well understood and associated harms wellknown. For pain lasting beyond the acute period, the prescribed use of strong opioids was, until recently, mainly restricted to cancer and palliative care, when life expectancy is limited. More recently, however, we have seen rapidly increasing use of both strong and weak opioids for chronic non-malignant pain. This has particularly been in North America where an "opioid epidemic" is apparent, but similar patterns are emerging across the world, including Europe.

The commonest problems with long-term use of opioids include constipation, nausea and sedation. Other, perhaps more serious problems include hyperalgesia, 